\title{
Prediction of Female diabetic Patient in India using different Learning Algorithms
}

\author{
Payal Bose ${ }^{1[0000-0002-2177-5775]}$, Prof. Samir K Bandyopadhyay ${ }^{1[0000-0002-4868-3459]}$ and Prof. Vishal Goyal ${ }^{1}$ \\ ${ }^{1}$ GLA University, Mathura-Delhi Road Mathura, Chaumuhan, Uttar Pradesh 281406
}

\begin{abstract}
Diabetics or Diabetic Mellitus is a metabolic disorder of blood sugar levels in the human body. It is a major non-communicable disease and involved many serious health risk issues. This disease is rapidly increasing in India. It is a chronic condition and it occurs when a body doesn't produce enough insulin hormone to control the blood sugar level. In this study, different variables have been analyzed that cause the diabetics, and different machine learning algorithms are used to predict whether an unknown sample is diabetes or not. For this purpose, PIMA diabetic detection for Female patients was used. Here 10 different classification model is used for prediction. Finally, the detailed performance analysis of the different variables of the PIMA dataset and also the classification model are discussed.
\end{abstract}

Keywords: Diabetic, Diabetic Mellitus, Diabetic Prediction, PIMA diabetic dataset, Female diabetic Patients, Machine Learning

\section{INTRODUCTION}

Diabetics Mellitus [1] or simple diabetes is a metabolic disorder or inadequate control of blood sugar levels. In the human body, the hormone insulin helps to move the glucose from blood to the cell and store the glucose as energy for future use. When the percentage of this glucose level is increases or decreases from its normal level, then diabetics are occurring. The high blood sugar level or the high diabetics can damage nerve, eyes, kidneys, skin, and many other organs. This is a very common disease and non-curable. But changing the lifestyle and food habits with treatment can help to maintain the blood sugar level and gives a healthy life. Diabetics come with depending on the different reasons.

a. Prediabetic. When the blood sugar level increases from normal to ceratin level but that should not enough to diagnose, then it is called Prediabetic [2]. It causes a heart attack and also the type-2 diabetics. Exercise and body weight maintenance can help to reduce the risk factors in the future.

b. Type-1 Diabetic. It is an insulin-dependent diabetic [3]. When the immune system of a body destroys the insulin-making cell then this type of diabetics is occurring. This type of diabetics usually occurs in children and young people. To control this diabetes a certain amount of insulin needs to consume on a daily purpose.

c. Type-2 Diabetic. This type of diabetes is insulin-independent [4]. It is commonly observed in adults but for the last 20 years this type of diabetes also common in the young generation due to overweight or obese. This diabetes occurs when an immune system resists to create the insulin hormone and the sugar level in blood is increased abruptly. Maintaining body weight, food habits, daily exercise, and proper medication can help to reduce the risk factors.

d. Gestational Diabetic. During pregnancy when some of the hormones resist to produce insulin hormones, then the blood sugar level in the mother's body is increasing. This type of diabetes [5] is often diagnosed during the middle or last stage of pregnancies. This type of diabetes is more riskier for the baby than the mother. Proper medication, enough nutrients can help to reduce the risk factors. 
Motivation and Aim of this study. Diabetes is one of the most common and serious health issues today. The occurrence of diabetic patients increasing day by day and most of them are female patients [6]. In several research it was observed that the factors like Body Mass Index, Blood Pressure, Insulin Level, Cholesterol are the main for causing diabetes. For female patients pregnancies is an additional but also an important factor. This study shows the behavior of the different key factors for diabetics patients and also the relationship between the main key factors. The aim of this study is to predict whether a patient is diabetic or not, particularly female patients, based on different machine learning algorithms.

\section{LITERATURE REVIEW}

In one research article, the authors made a comparative analysis of different machine learning algorithms. They evaluated the performance of the different machine learning algorithms using the PIMA diabetics dataset of female patients in India. They show the random forest classifier gives more than $74 \%$ accuracy [7].

The authors in their study used the PIMA diabetics dataset with the other dataset collected from Kurmitola General hospital in Bangladesh. They used different machine learning algorithms to perform the prediction. Finally shows the Naïve Bayes algorithm gives the best result among them [8].

Other authors used this PIMA diabetics dataset in their study to predict the disease. They also used the different machine learning algorithms to calculate the classification accuracy, precision, F1-score, and accuracy under the ROC curves [9].

In one research article, the authors investigate the prediction of diabetics based on the input features FPG and HbA1c. They used five different machine learning algorithms with hierarchical clustering, feature elimination, and feature permutation techniques. They identify different risk factors that are indirectly involved with diabetes classification [10].

The authors in their study used diverse machine learning algorithms on the PIMA diabetic prediction dataset. They used the classifiers Artificial Neural Network (ANN), Naive Bayes (NB), Decision Tree (DT), and Deep Learning (DL) and achieved $90 \%$ to $98 \%$ accuracy. They also show that the deep learning approach achieved the maximum accuracy, $98.04 \%$ [11].

\section{DATASET OVERVIEW}

\section{a. DATASET}

The PIMA diabetic dataset[12] of Indian female patients was downloaded from Kaggle. This dataset was originally collected from the National Institute of Diabetes and Digestive and Kidney Diseases. The purpose of this dataset is to diagnosis whether a patient is diabetic or not based on certain measurement parameters. All the patients in this dataset are female and at least 21 years old of Pima Indian heritage.

\section{b. DATASET DETAILS}

This dataset [13] contains a total of 768 data of female patients. Among them, 500 female patients are diagnosed as non-diabetic and 268 female patients are diabetic. The diagnosis result is stored as the binary values in the dataset for each patient with the other attributes. Table 1 and figure 1 show the dataset summary and the diagnosis details of the PIMA dataset. 
Table 1: PIMA Dataset Summary

\begin{tabular}{|l|c|}
\hline \multicolumn{2}{|c|}{ PIMA Diabetic Detection dataset } \\
\hline Total No of Observation & 768 \\
\hline Total No of Features & 9 \\
\hline Total No of Diabetic Patients & 268 \\
\hline Total no of Non-diabetic patients & 500 \\
\hline Diabatic : Non-Diabatic (ratio) & $0.35: 0.65$ \\
\hline
\end{tabular}

c. DATASET FEATURE DETAILS

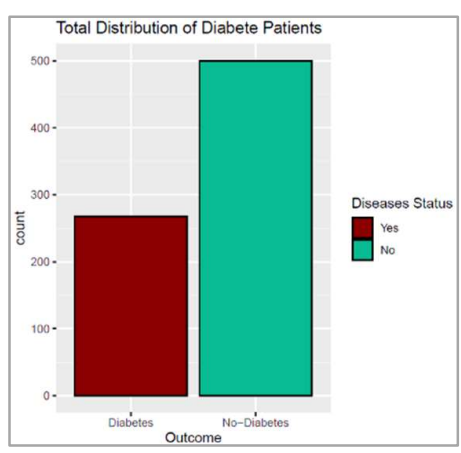

Figure 1:Total no of Diabatic Patient Distribution

The dataset has 8 predictive variables and one responsive variable. The variables' details are shown in table 2 .

Table 2: Dataset Variables Details

\begin{tabular}{|l|l|l|l|l|}
\hline No. & \multicolumn{1}{|c|}{ Feature name } & \multicolumn{1}{c|}{ Feature Details } & \multicolumn{1}{c|}{$\begin{array}{c}\text { Min } \\
\text { Value }\end{array}$} & \multicolumn{1}{c|}{$\begin{array}{c}\text { Max } \\
\text { value }\end{array}$} \\
\hline 1 & Pregnancies & Number of times pregnant & 0.0 & 17.0 \\
\hline 2 & Glucose & $\begin{array}{l}\text { Plasma glucose concentration 2 hours in an oral } \\
\text { glucose tolerance test }\end{array}$ & 0.0 & 199.0 \\
\hline 3 & BloodPressure & Diastolic blood pressure $(\mathrm{mm} \mathrm{Hg})$ & 0.0 & 122.0 \\
\hline 4 & SkinThickness & Triceps skin fold thickness $(\mathrm{mm})$ & 0.0 & 99.0 \\
\hline 5 & Insulin & 2-Hour serum insulin $(\mathrm{mu} \mathrm{U} / \mathrm{ml})$ & 0.0 & 846.0 \\
\hline 6 & BMI & Body mass index (weight in kg/(height in m) $\left.)^{2}\right)$ & 0.0 & 67.1 \\
\hline 7 & DiabetesPedigreeFunction & Diabetes pedigree function & 0.078 & 2.42 \\
\hline 8 & Age & In years & 21 & 81 \\
\hline 9 & Outcome & Class variable $(0$ or 1$)$ & - & - \\
\hline
\end{tabular}

\section{d. DATASET ANALYSIS}

1. Pregnancies. The dataset contains a total of 8 predictive variables. All the patients in this dataset are female so the no of pregnancies is one of the most important variables for analysis. Figure 2a shows the boxplot of no of pregnancies of diabetic and non-diabetic female patients. Figure 3 a shows the bar plot of diabetic and non-diabetic patients grouped by the number of time pregnancies of female patients.

2. Glucose. Variable glucose is another important predicting variable in this dataset. Here the glucose concentration level is observed after 2 hours of a meal. Generally, the glucose concentration level for a normal person is up to 140 after 2 hours of a meal. The percentage bar plot shows the concentration level of below 140 and above 140 of the female patients. Figure $2 \mathrm{~b}$ and $3 \mathrm{~b}$ shows the boxplot and bar plot of glucose concentration level.

3. Blood Pressure. Here the blood Pressure level is divided into three categories 1) below $65 \mathrm{~mm}$ hg is called lownormal, 2) 65-89 range is called normal and 3) above 89 is higher than normal or Hypertension. Figure 2c shows the boxplot of the blood pressure level of female diabetes and non-diabetes patients and $3 \mathrm{c}$ show the percentage of diabetes and non-diabetes patients based on the blood pressure level. 
4. Skin Thickness. Human skin thickness is determined by collagen. It is produced underneath the skin. Depending on the insulin level the skin thickness is determined. Figure $2 \mathrm{~d}$ shows the boxplot of the female patients and $3 \mathrm{~d}$ shows the percentage level of skin thickness of the female diabetic and non-diabetic patients.

5. Insulin. It is a hormone that keeps balance the blood sugar level in the human body. After 2 hours of consuming a meal, the normal insulin level is less than $200 \mathrm{mu} \mathrm{U} / \mathrm{ml}$. Figure 2e shows the box plot of the insulin level of diabetic and non-diabetic female patients and $3 \mathrm{e}$ shows the percentage level.

6. BMI (Body Mass Index). This variable is used to measure body fat. For a diabetic patient, this is an important measuring parameter. Figures $2 \mathrm{f}$ and $3 \mathrm{f}$ show the box plot and the percentage level of BMI of the female diabetic and non-diabetic patients.

7. Diabetic Pedigree Function (DPF). It is a parameter that gives the report about the patient's family diabetic history. This function provides the relationship between the genetic and non-genetic relatives' diabetic status. Figure $2 \mathrm{~g}$ and $3 \mathrm{~g}$ show the box plot and the percentage level of DPF of the female diabetic and non-diabetic patients.

8. Age. Age is one of the most important parameters for female patients w.r.t diabetic. Based on the different age groups the value of diabetic predicted parameters is changed. Figure $2 \mathrm{~h}$ and $3 \mathrm{~h}$ shows the box plot and the percentage level of the different Age groups of the female diabetic and non-diabetic patients.
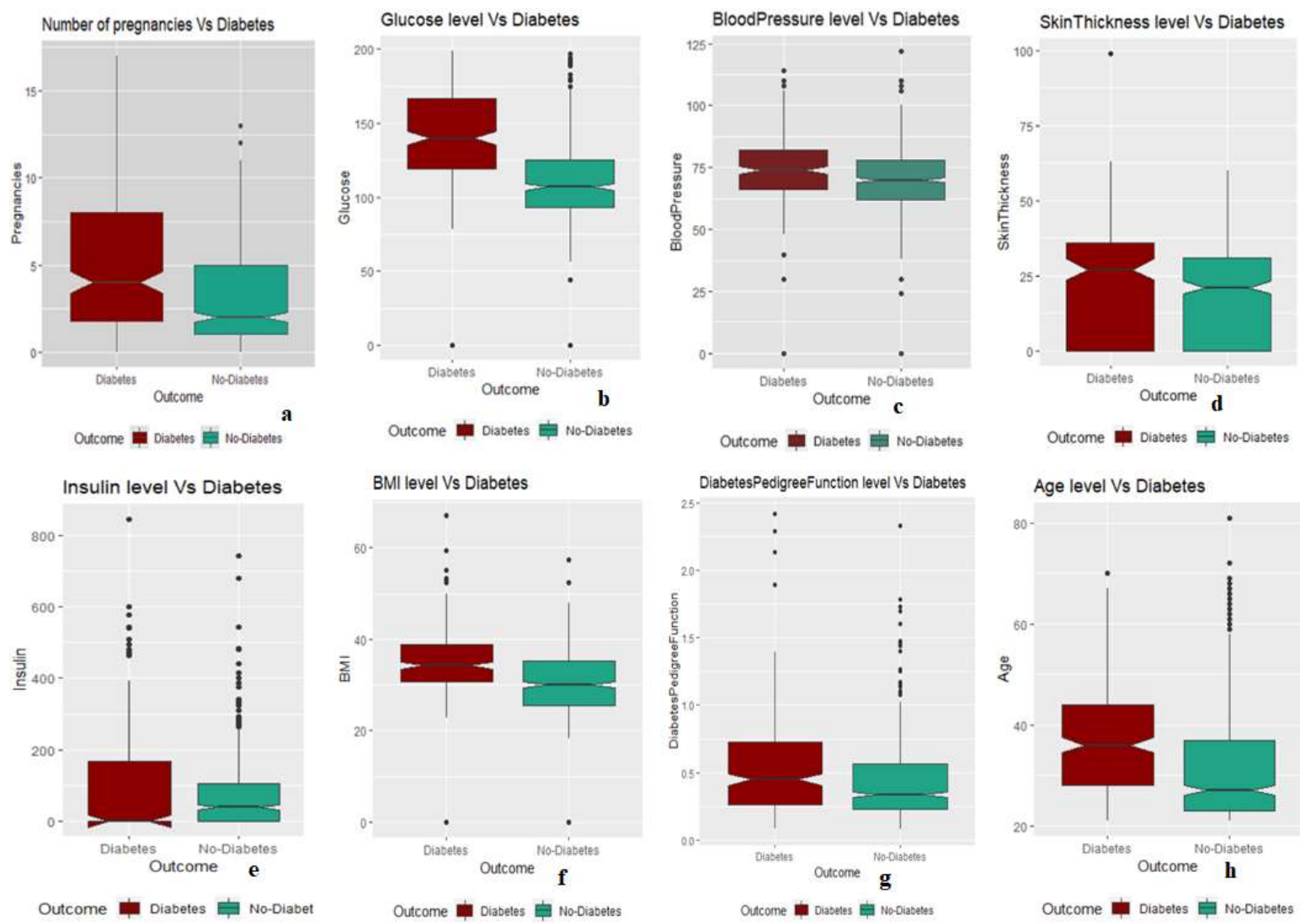

Figure 2 (a-h): Boxplot of different predictive variables of Female Patients 

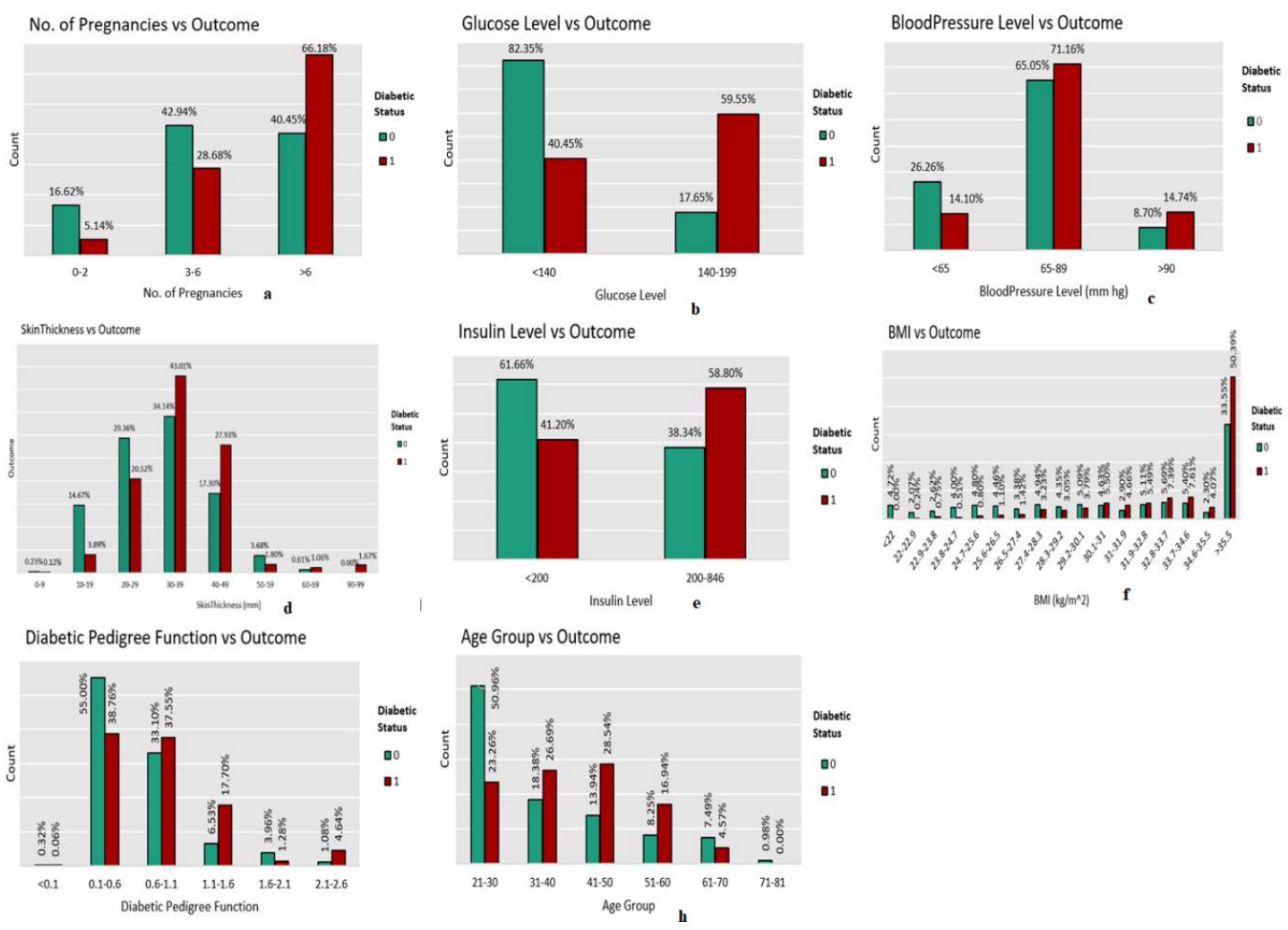

Figure 3 (a-h): Percentage Bar plot of different predictive variables of Female Patients

\section{e. DATASET CORRELATION}

A correlation matrix is used to explain the statistical relationship between the variables. Figure 4 shows the correlation of the 8 predictive variables and 1 responsive (Outcome) variable of the PIMA diabetics dataset of female patients in India.

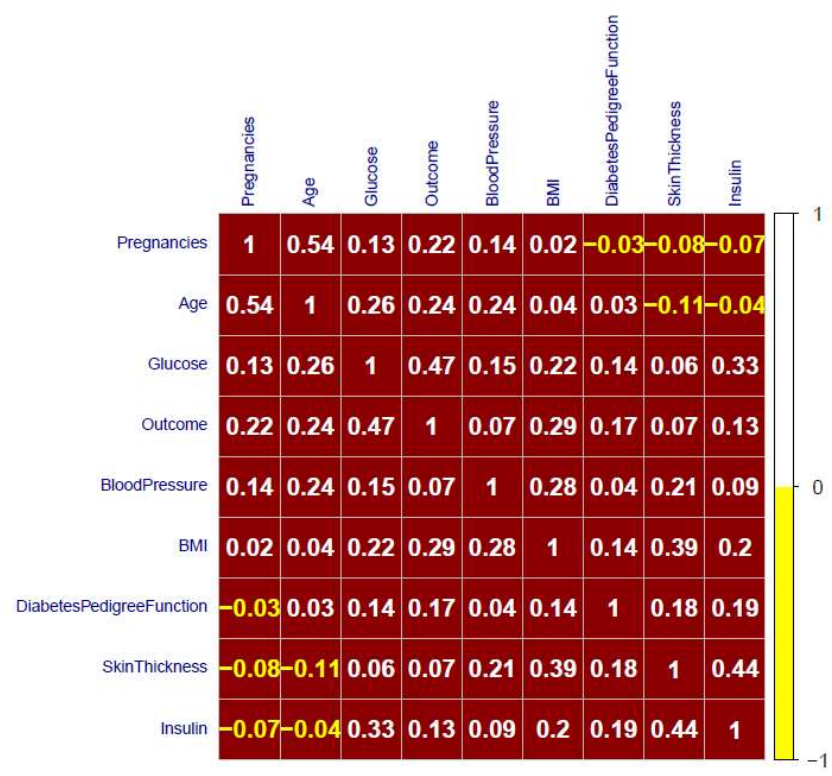

Figure 4: Correlation Plot of PIMA Diabetics Dataset 
From the correlation matrix plot, it is observed that the dataset has five important predictive variables. They are, 1) No. of Pregnancies, 2) Glucose level, 3) Insulin level, 4) BMI (Body Mass Index), and 5) Age group. Again, the BMI level also correlated with two other variables 1) Skin Thickness, and 2) Blood Pressure level. The relationship among all the variables is depicted in figure $5(\mathrm{a}-\mathrm{d})$.
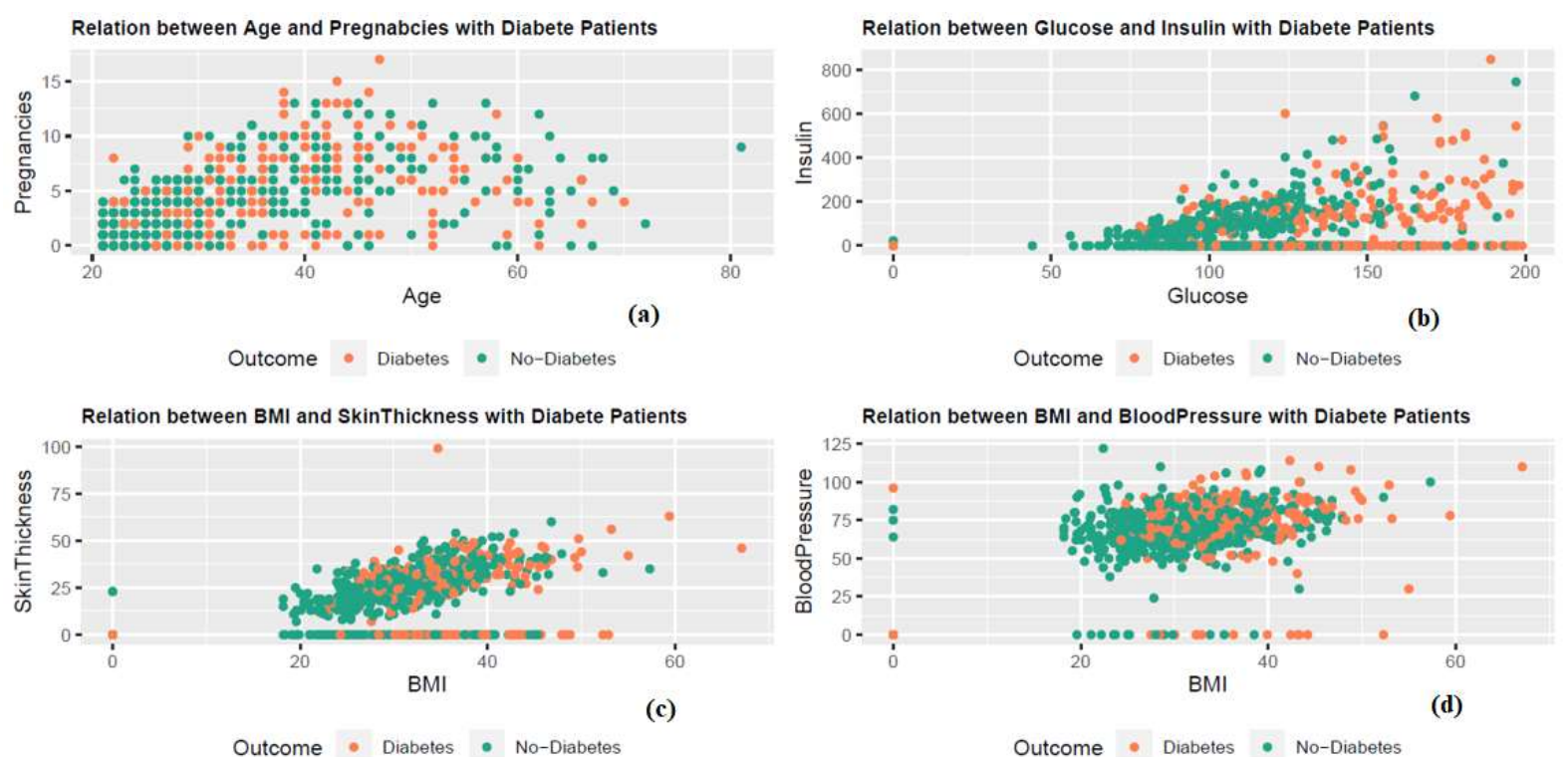

Figure 5(a-d): The relation between the important variables for diabetic prediction

\section{METHODOLOGY}

The process to predict a female patient diabetic or not is depicted in figure 6. For this study, 10 different machine learning algorithms are used to check the prediction rate. First, the dataset was pre-processed to be used for the machine learning algorithms. Then the dataset split into training and testing sets. After that, the algorithms are applied to the training set to create the trained dataset. Finally, the trained dataset is applied to the test set to predict the outcome.

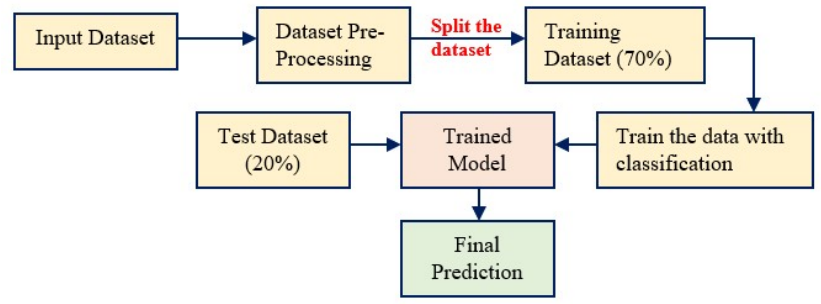

Figure 6: Workflow of the model

1. Data Pre-processing. In this part, it is needed to check if any data missing in the original dataset or not. If any data is missing then replaced the null data with the mean values of that variable. After filling in the missing data, the dataset is finally ready for the classification algorithm.

2. Split the dataset. After pre-processing the dataset is divided into two parts, 1) Training Dataset to create a Trained Model, 2) Test Dataset. For training the $80 \%$ data and for testing $20 \%$ data in the whole dataset is used.

3. Classification Model. For the prediction of the outcome, in this experiment 10 classification algorithms are used. They are 1) Decision Tree, 2) Random Forest, 3) Gradient Boosting, 4) Logistic Regression, 5) Linear Regression, 
6) Support Vector Machine, 7) Discriminant Analysis, 8) Quadratic Discriminant Analysis, 9) Mixed Discriminant Analysis and 10) K-Nearest Neighbour Algorithm.

\section{EXPERIMENTAL RESULT}

All classifications result shown in table 3. The performance of all the classifiers was analyzed based on the training, testing accuracy. All the accuracy is calculated using the below formula.

$$
\text { Accuracy }=\frac{T_{P}+T_{N}}{T_{P}+F_{P}+T_{N}+F_{N}}
$$

Where, $T_{P}=($ True positive $)=$ Actual Positive Class and Positive Prediction, $T_{N}=($ True Negative $)=$ Actual Positive Class and Negative Prediction, $F_{P}=($ False Positive $)=$ Actual Negative Class and Positive Prediction, $F_{N}=($ False Negative $)=$ Actual Negative Class and Negative Prediction.

Table 3: Performance Analysis of all the Classifiers

\begin{tabular}{|c|c|c|}
\hline Methods & Training Accuracy & Testing Accuracy \\
\hline Decision Tree & 0.8093645 & 0.7470588 \\
\hline Random Forest & 1.0000000 & 0.8176471 \\
\hline Gradient Boosting & 0.8227425 & 0.7117447 \\
\hline Logistic Regression & 0.7909699 & 0.7294118 \\
\hline Linear Regression & 0.7742475 & 0.7176471 \\
\hline Support Vector Machine & 0.7575251 & 0.8352941 \\
\hline Discriminant Analysis & 0.7491639 & 0.8000000 \\
\hline Quadratic Discriminant Analysis & 0.7419963 & 0.8000000 \\
\hline Mixed Discriminant Analysis & 0.7591973 & 0.8176471 \\
\hline K-Nearest Neighbour & 0.7956429 & 0.8000000 \\
\hline
\end{tabular}

\section{CONCLUSION}

In this study, PIMA diabetic detection dataset was used to predict diabetics in female patients. A detailed analysis of 8 predictive variables and one responsive variable was done in this study. After that, a correlation matrix was calculated to show the important variables and their dependencies variable. Various supervised machine learning algorithms are used to calculate the prediction performance. Finally, a detailed comparative analysis of the algorithms is also performed. Here only one dataset is used and the number of observations in this dataset is quite small. Therefore, the future work is to use a large dataset and also apply the deep learning algorithms for better performance.

\section{ACKNOWLEDGMENTS}

Smith, J.W., Everhart, J.E., Dickson, W.C., Knowler, W.C., \& Johannes, R.S. (1988). Using the ADAP learning algorithm to forecast the onset of diabetes mellitus. In Proceedings of the Symposium on Computer Applications and Medical Care (pp. 261--265). IEEE Computer Society Press. 


\section{REFERENCES}

[1] MedicalNewsToday. What to Know about PCOS and Diabetes. Available online: https://www.medicalnewstoday.com /articles/326185 (accessed on 29 August 2020).

[2] Watson, Stephanie. "Diabetes: Symptoms, Causes, Treatment, Prevention, and More." Healthline, Healthline Media, 27 Feb. 2020, www.healthline.com/health/diabetes.

[3] IDF. Type 1 Diabetes. Available online: https://www.idf.org/aboutdiabetes/type-1-diabetes.html (accessed on 20 January 2020).

[4] IDF Diabetes Atlas, A.D. Type 2 Diabetes. Available online: https://www.idf.org/aboutdiabetes/type-2diabetes.html (accessed on 20 March 2020).

[5] IDF. Gestational Diabetes. Available online: https://www.idf.org/our-activities/care-prevention/gdm.html (accessed on 1 June 2020).

[6] WHO. Diabetes. Available online: https://www.who.int/news-room/fact-sheets/detail/diabetes (accessed on 1 June 2020).

[7] Varma, K.M.; Panda, D.B.S. Comparative analysis of Predicting Diabetes Using Machine Learning Techniques. J. Emerg. Technol. Innov. Res. 2019, 6, 522-530.

[8] Pranto, B., Mehnaz, S. M., Mahid, E. B., Sadman, I. M., Rahman, A., \& Momen, S. (2020). Evaluating machine learning methods for predicting diabetes among female patients in Bangladesh. Information (Switzerland), 11(8). https://doi.org/10.3390/INFO11080374

[9] Kumar, S., Kumar, S., Kumar, K., \& Abhisekh, P. A. (2021). Prediction of Diabetes in Females of Pima Indian Heritage : A Complete Supervised Learning Approach. 12(10), 3074-3084.

[10] Ahmad, H. F., Mukhtar, H., Alaqail, H., Seliaman, M., \& Alhumam, A. (2021). Investigating health-related features and their impact on the prediction of diabetes using machine learning. Applied Sciences (Switzerland), 11(3), 1-18. https://doi.org/10.3390/app11031173

[11] Naz, H., \& Ahuja, S. (2020). Deep learning approach for diabetes prediction using PIMA Indian dataset. Journal of Diabetes and Metabolic Disorders, 19(1), 391-403. https://doi.org/10.1007/s40200-020-00520-5

[12] PIMA. University of California, Irvine Learning Repository. Available online: https://www.kaggle.com/uciml/pima-indians-diabetes-database (accessed on 6 October 2020).

[13] Smith, J.W., Everhart, J.E., Dickson, W.C., Knowler, W.C., \& Johannes, R.S. (1988). Using the ADAP learning algorithm to forecast the onset of diabetes mellitus. In Proceedings of the Symposium on Computer Applications and Medical Care (pp. 261--265). IEEE Computer Society Press. 